\title{
CYCLIC TEXTURES IN ALUMINIUM WIRES
}

\author{
U. SCHLÄFER and H. J. BUNGE \\ Zentralinstitut für Festkörperphysik und Werkstofforschung, \\ Dresden, der Deutschen Akademie der Wissenschaften zu Berlin, G.D.R.
}

(Received December 19, 1971)

\begin{abstract}
Three-dimensional orientation distribution functions were calculated from neutron diffraction pole figures of unwound cylinders taken at different distances from the centre of cold drawn Al-wires. Their features change from the axially symmetric type at the very centre of the wire towards a texture near to the rolling type at the surface. Relations between the three-dimensional function and ordinary fibre texture pole figures were used to study the dependence of the textures on certain processing variables for cold drawn as well as recrystallized wires.
\end{abstract}

\section{INTRODUCTION}

Because of the geometrical conditions of the drawing process the texture of a drawn wire exhibits axial symmetry. This means that crystals the orientations of which result from one another by a rotation about the wire axis occur with equal frequency. Hence the orientation distribution function is independent of this angular coordinate. Essentially it is a two-dimensional function namely the inverse pole figure as compared to the case of general textures which needs a three-dimensional distribution function for complete description. In this sense wire textures are much simpler, as for example sheet textures, because the whole problem is reduced by one dimension.

The above considerations are valid if one looks at the orientations of the crystals only and neglects their positions in space, in other words if one treats the wire as infinitely thin. If, however, one takes the finite dimensions of a wire into account then the geometry of the drawing process requires only the central axis of the wire to be an axis of rotation. There is no need for an off-central line to be an axis of symmetry with respect to the orientation distribution function of the crystallites positioned at this line. The central axis of rotational symmetry does require only that the texture is the same at cylindrical tubes coaxial with the wire axis and this only if we refer the crystal orientations to a coordinate system made up of radial, tangential, and axial directions. Of course, crystals having the same orientation in this coordinate system need not to be parallel at all. They become parallel, however, if the cylindrical surface is unwound into a plane. Because of the geometry of the drawing process the plane containing the wire axis and the radial direction must be a mirror plane, but no further symmetry conditions are required. Thus the unwound cylindrical surfaces may be compared with offcentral planes parallel to the rolling plane of sheet materials. Because of the necessary unwinding process, however, wire textures in this sense must be regarded as even more complicated than sheet textures. In this approach they need a threedimensional orientation distribution function in order to be fully described, and this function, furthermore, may depend on the distance from the wire axis.

Investigations of wire textures including the unwinding process have been carried out for the first time by Stüwe and coworkers ${ }^{1,2}$. It was shown that for off-central layers the wire-axis direction was not an axis of rotational symmetry. Hence these textures were no ordinary fibre textures. The radial and the tangential directions were not equivalent with respect to the orientation distribution of the crystallites. Textures of this type were called cyclic textures. They showed some resemblance to rolling textures, if the wire axis was compared with the rolling direction and the tangential plane with the rolling plane. If one proceeds from the surface towards the wire axis, however, the cyclic textures must change into ordinary fibre textures because of the symmetry requirements of the wire axis. Hence cyclic textures may be regarded as occupying an intermediate position between rolling textures and ordinary fibre textures. Since attempts of rationalizing rolling textures in terms of pole figures and ideal orientations only proved insufficient this was 
to be expected the more so for cyclic textures. Hence in the present investigation, pole figures taken from unwound cylinders were used to calculate threedimensional orientation distribution functions the same way as was formerly done with sheet textures. The measurements were carried out by neutron diffraction because of the much lower level of experimental errors of this method as compared with $\mathrm{X}$-ray diffraction. Once the essential features of the three-dimensional orientation distribution function are known, its certain parameters may be related to parameters immediately taken from pole figures. This enables the dependence of textures on certain processing variables to be studied without the expensive calculation of the three-dimensional orientation distribution function for each specimen.

\section{EXPERIMENTAL}

The material used was aluminium $99.5 \%$ pure containing the following impurities: $0.01 \% \mathrm{Mg}, 0.01 \%$ $\mathrm{Mn}, 0.11 \% \mathrm{Si}, 0.22 \% \mathrm{Fe}$. An extruded rod $22 \mathrm{~mm}$ in diameter was cold drawn unidirectionally to $4 \mathrm{~mm}$ which corresponds to $97 \%$ reduction in area. Out of these wires small tubes, the dimensions of which are given in Table $I$, were made by drilling and lathing. They were slotted and subsequently flattened into small plates. Naturally this

TABLE I

Dimensions of the specimens used for neutron-diffraction measurements.

\begin{tabular}{cccccc}
\hline & $\begin{array}{c}\text { Outer } \\
\text { diameter } \\
(\mathrm{mm})\end{array}$ & $\begin{array}{c}\text { Inner } \\
\text { diameter } \\
(\mathrm{mm})\end{array}$ & $\begin{array}{c}\text { Wall } \\
\text { thickness } \\
(\mathrm{mm})\end{array}$ & $\begin{array}{c}\text { Length } \\
(\mathrm{mm})\end{array}$ & Quantity \\
\hline Al 1 & 4 & 3 & 0.5 & 30 & 30 \\
A1 2 & 3 & 2 & 0.5 & 20 & 60 \\
Al 3 & 2 & 1 & 0.5 & 20 & 80 \\
Al 4 & 1 & - & - & 20 & 60 \\
\hline
\end{tabular}

imposed an additional plastic deformation which was, however, regarded as negligible with respect to the preceding deformation. These plates were too small for neutron diffraction measurements, therefore, several of them were assembled to form a composed specimen of about 40 by 40 by $1 \mathrm{~mm}$. Removing the machined surface was not necessary because neutron diffraction is essentially a volume effect. Thus the thin distorted layer does not contribute to the reflected intensity at any noticeable extent. The neutron diffraction measurements were carried out at the Rossendorf research reactor using a semi-automatic texture device described by Tobisch, Betzl, and Reichel ${ }^{3}$ which was mounted on the neutron diffractometer described by Betzl and Kleinstück ${ }^{4}$. The experimental procedure followed that given by Kleinstück and Tobisch ${ }^{5}$. The intensity of the reflected neutron beam was measured for distinct angular positions of the specimen in the back reflection and transmission range forming a $6^{\circ}$ grid. The intensity output figures were immediately punched into tape. By means of a computer programme the values were corrected for background, absorption, and geometrical factors, thus yielding quantitative pole density values normalized in times random units. (The normalization was carried out by the computer too.) Drawings of the pole figures were prepared automatically using a computer-operated plotter which was programmed to construct the intersections of the intensity-level lines with the coordinate lines. ${ }^{8} \mathrm{Up}$ to ten level lines could be indicated by different marks; the intensity levels could be chosen arbitrarily. An example of a computer-plotted pole figure is shown in Figure 1. Because of the limitations of our present computer programmes specimen symmetries lower than orthorhombic could not be handled.

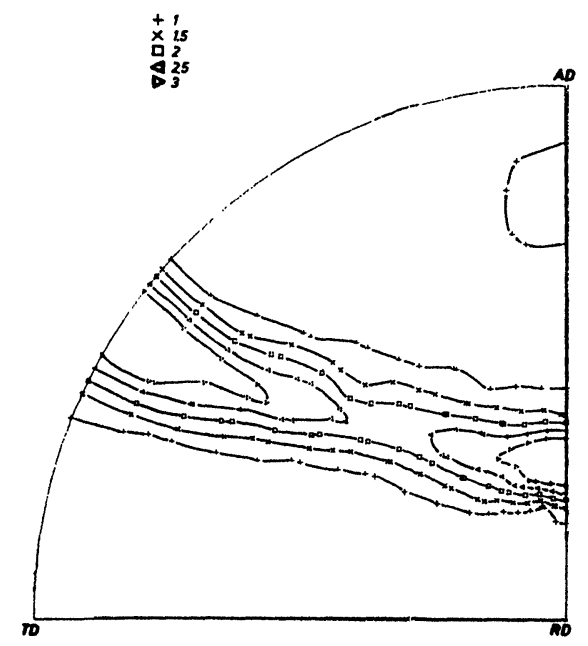

FIGURE 1 Computer plotted pole figure (the marks corresponding to one level line were connected by hand).

In particular this means drawing- and counterdirections could not be distinguished in the subsequent mathematical procedure. Hence the neutron diffraction specimens were assembled without reference to drawing- and counter-directions, thus introducing an additional artificial symmetry. 
Accordingly pole figure values were measured only for one quadrant of the pole figure (the other quadrants being the same within the limits of experimental error). We are fully aware that this is a restriction and it is intended to abolish it in a further investigation.

Four pole figures were taken for each specimen, namely the (111), (200), (220) and (311). These were used later on to calculate three-dimensional orientation distribution functions. The specimens labeled $\mathrm{Al} 4$ representing the central part of the wires were treated as ordinary fibre textures. They were assembled in three parallel layers to form a composed plate of 20 by $20 \mathrm{~mm}$ which was measured in the symmetric transmission position only, the angular steps being $2^{\circ}$. The same four pole figures mentioned above were taken, and subsequently the inverse pole figure was calculated.

Additional measurements were carried out with thin aluminium wires ranging from $0.04 \mathrm{~mm}$ to $1 \mathrm{~mm}$ in diameter. Four different materials were used, the compositions of which are shown in Table II. The

\section{TABLE II}

Compositions of the four aluminium wires used for X-ray investigations (in wt.- $\%$ ).

\begin{tabular}{lllllll}
\hline Charge & $\mathrm{Cu}$ & $\mathrm{Si}$ & $\mathrm{Mg}$ & $\mathrm{Fe}$ & $\mathrm{Zn}$ & $\mathrm{Al}$ \\
\hline I & 0.01 & 0.31 & - & 0.28 & - & 99.4 \\
II & 0.01 & 0.12 & 0.04 & 0.28 & 0.05 & 99.5 \\
III & 0.006 & 0.014 & - & 0.018 & - & 99.96 \\
IV & 0.01 & 0.12 & 0.02 & 0.14 & 0.02 & 99.7 \\
\hline
\end{tabular}

charges I, II and IV were of commercial purity $(99.5 \%$ ) whereas III was $99.9 \%$ pure. They were cold drawn from $1 \mathrm{~mm}$ to a maximum deformation of $99.9 \%$ reduction in area corresponding to $0.1 \mathrm{~mm}$ in diameter starting from the recrystallized state. These wires were investigated with $\mathrm{X}$-ray fibre texture methods using $\mathrm{MoK}_{\alpha}$-radiation. A large number of wires were placed parallel to one another forming something like a sheet which was investigated in the symmetric transmission position. For the sake of homogenization the sheet was translated in two perpendicular directions in its own plane. The reflected intensity was measured in $1^{\circ}$-steps using a special step-operated specimen device $^{6}$ mounted on the X-ray goniometer HZG1 from VEB Freiberger Präzisionsmechanik. For diameters below $0.18 \mathrm{~mm}$ absorption was neglected, whereas the wires of larger diameter were embedded in an aqueous solution of strontium chloride having the same absorption coefficient as aluminium ${ }^{7}$ thus forming a plane-parallel plate of homogeneous absorption. In this way all absorption corrections could be avoided. Some of the specimens were annealed after deformation at temperatures up to $500^{\circ} \mathrm{C}$. The annealings were carried out in a tube furnace in normal air atmosphere. For further experimental details see Ref. 9.

\section{RESULTS}

In Figure 2 the neutron diffraction pole figures corresponding to unwound cylinders of different diameters are shown. They were mainly used as raw material for the calculation of the complete (three-dimensional) orientation distribution functions. As is well-known the complete orientation of a crystal in a polycrystalline material must be specified by at least three parameters which may be chosen, for example, as the Euler angles. Let a crystal originally be in a position with its axes parallel to the specimen coordinate axes (in the present case $x=$ axis direction, $y=$ tangential direction, $z=$ radial direction, Figure 3 ). Then it is rotated successively about its $z$-axis through the angle $\varphi_{1}$, about its $x$-axis in its new position through $\phi$, and finally once more about the new $z$-axis through $\varphi_{2}$. The final orientation of the crystal is described then by the three Euler angles $\varphi_{1}, \phi, \varphi_{2}$. The threedimensional orientation distribution function gives the relative frequency of crystals having the orientations described by $\varphi_{1}, \phi, \varphi_{2}$, measured in multiples of the random distribution. This function was calculated from the pole figures following the methods described elsewhere. ${ }^{10}$ The series was extended up to $l=22$ and the results were obtained in $5^{\circ}$-steps for the variables $\varphi_{1}, \phi, \varphi_{2}$; they were automatically plotted in a similar way as were the pole figures. In Figure 4 they are shown for the three different cylinder diameters. If one imagines the different $\varphi_{2}$-sections stacked one upon another, one easily recognizes that the ranges of high orientation density form something like a tube in the orientation space. If one considers only the points of maximum orientation density, these form a line which may be called the skeleton line of the orientation distribution. The orientations of the skeleton line may be transferred into the inverse pole figure. This means the orientation of the wire axis direction or radial direction may be given with respect to the crystallographic axes, represented in stereographic projection. This is shown in Figure 5. The skeleton line is the same for the three different cylinder 
(111)
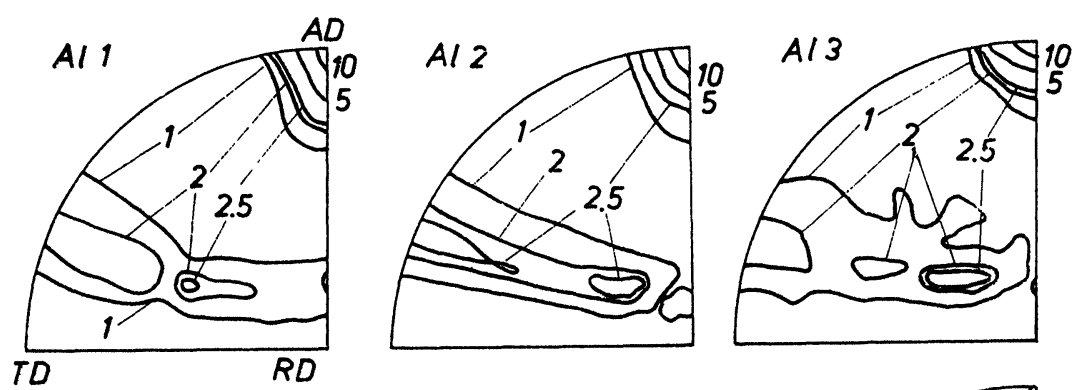

(200)
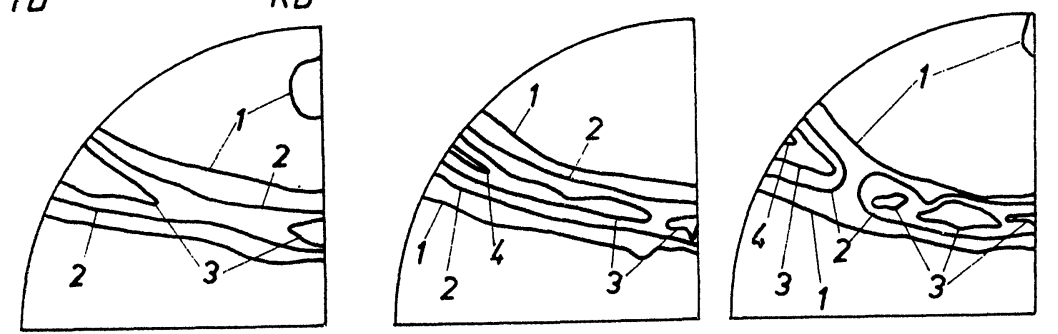

(220)
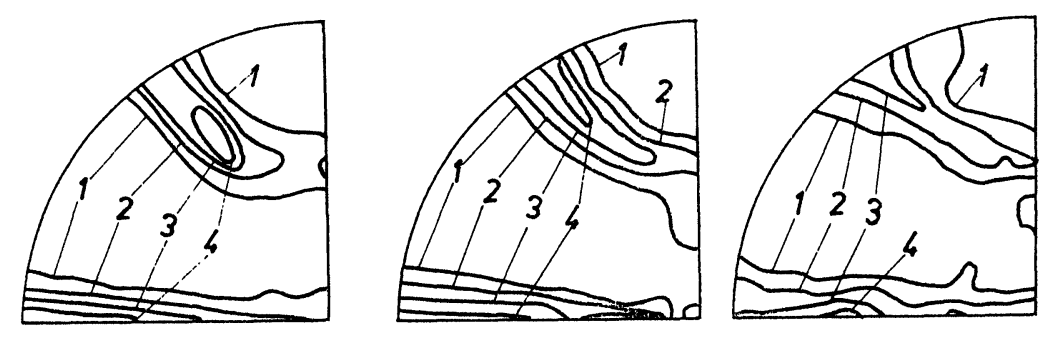

(391)
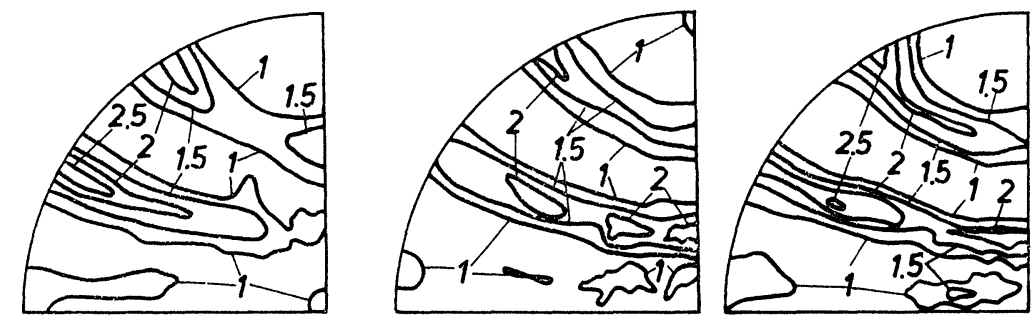

FIGURE 2 Neutron diffraction pole figures of unwound cylinders of different diameters taken from an aluminium wire cold drawn $97 \%$. (Multiples of the random distribution) $A D=$ axis direction, $R D=$ radial direction, $T D=$ tangential direction.

diameters and it corresponds to a fibre texture with (111) parallel to the fibre axis.

If the texture were a true fibre texture the orientations related to one another by a rotation about the fibre axis ought to be equally frequent, in other words, the orientation density along the skeleton line ought to be constant. This is clearly not the case as is seen in Figure 6. For explanation it must be mentioned that the volume of orientation space represented in Figure $4\left(0^{\circ} \leqq \varphi_{1}, \phi, \varphi_{2} \leqq 90^{\circ}\right)$ contains three symmetrically equivalent subranges connected to one another by the three-fold axis of the cubic crystal lattice. These three subranges are clearly seen in Figure 5. In Figure 6 their boundaries are indicated by heavy vertical lines at $25^{\circ}$ and $45^{\circ}$. It is seen that two intensity maxima occur at orientations having a [101] or [112] direction parallel to the radial direction. In the outermost cylinder (A1 1) a third orientation with [123] parallel to the radial direction was found although in this case the differences in orientation density were very small. According to Figure 7 the amount of the different cyclic components (components with preferred radial orientations) and the ordinary 


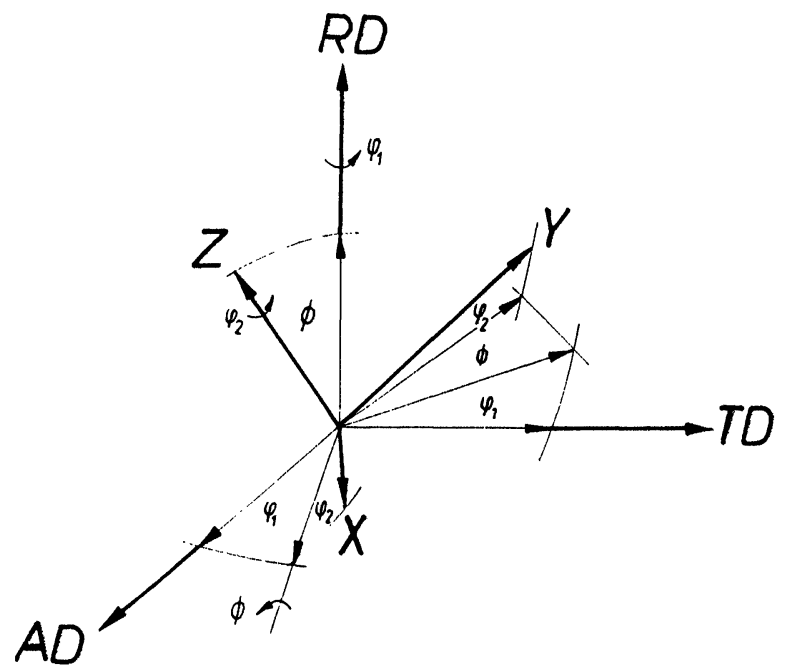

FIGURE 3 Definition of the Euler angles in the case of cyclic textures.

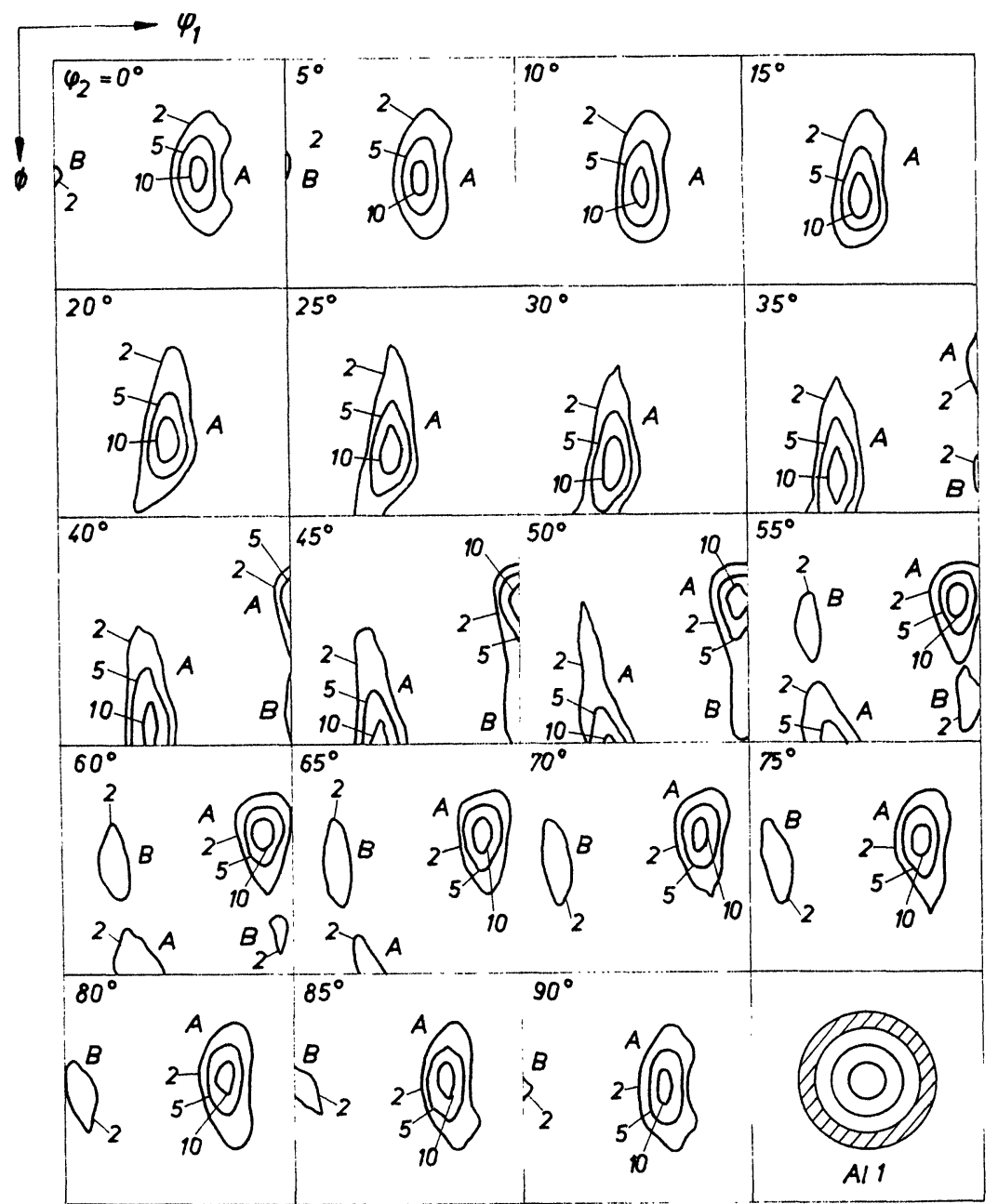

FIGURE 4a 


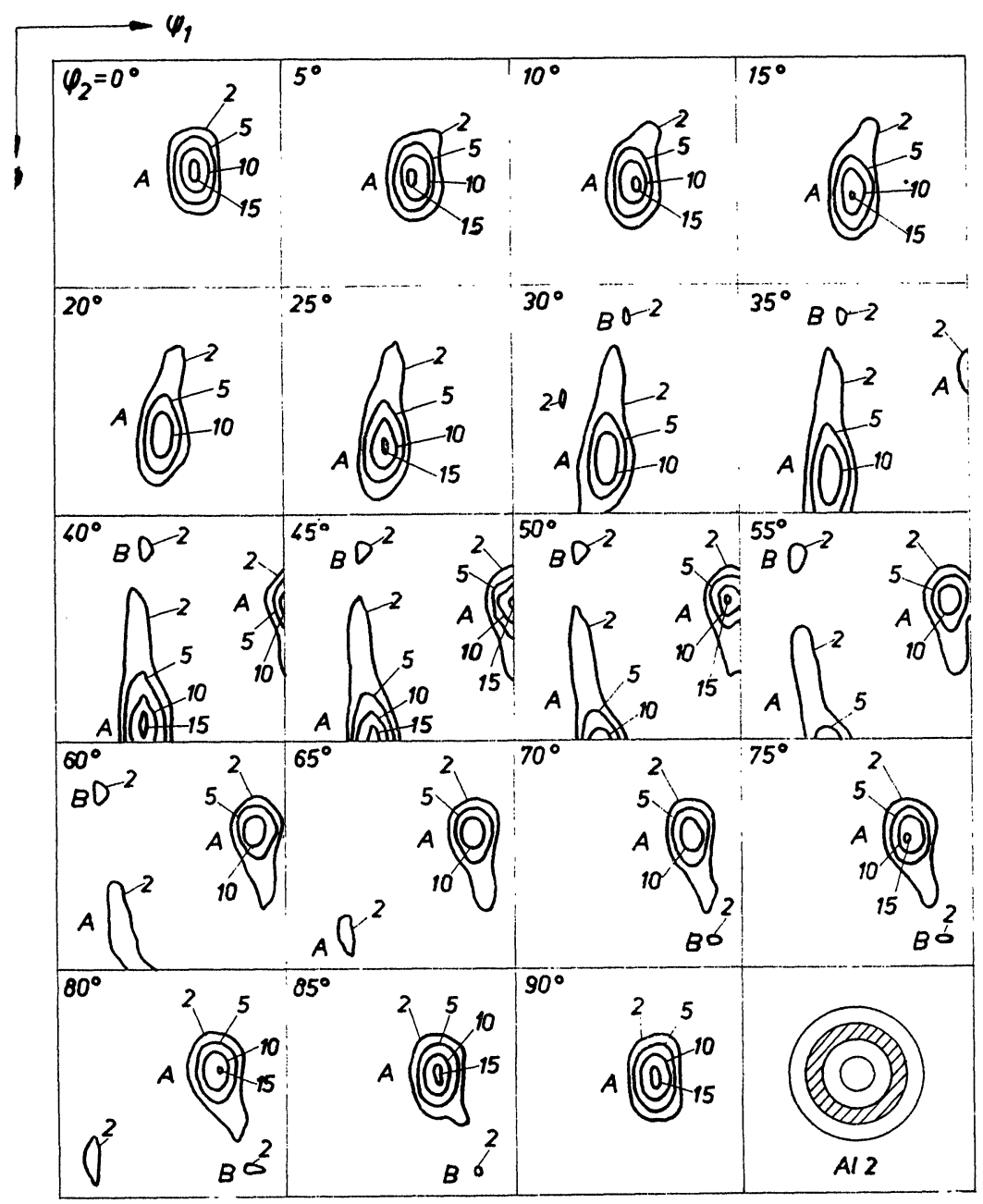

FIGURE $4 b$

fibre texture may be estimated. The results are summarized in Table III.

TABLE III

Relative amount of ordinary and cyclic fibre texture components.

\begin{tabular}{|c|c|c|c|c|c|}
\hline & $\begin{array}{c}\text { Ordinary } \\
\text { fibre } \\
\text { texture } \\
(\%)\end{array}$ & $\begin{array}{c}(112)[111] \\
(\%)\end{array}$ & $\begin{array}{c}(101)[111] \\
(\%)\end{array}$ & $\begin{array}{c}(21 \overline{3})[111] \\
(\%)\end{array}$ & $\begin{array}{c}\text { Cyclic } \\
\text { textures } \\
\text { total } \\
(\%)\end{array}$ \\
\hline Al 1 & 90.3 & 3.5 & 1.2 & 5.0 & 9.7 \\
\hline $\mathrm{Al} 2$ & 92.6 & 1.8 & 5.5 & - & 7.4 \\
\hline Al 3 & 82.3 & 3.7 & 13.9 & - & 17.7 \\
\hline
\end{tabular}

Another remarkable feature of the orientation distributions of Figure 4 is the form of the spread about the skeleton line. For the innermost cylinder it is more circularly shaped whereas for the outer layers directions of smaller and larger spread may be distinguished. In the upper part of Figure 8 these directions are indicated for the $\varphi_{2}=0$ section of $\mathrm{Al} 1$. The lower part of this figure shows the form of spread along these directions. With the scales used the spread is represented by straight lines corresponding to Gaussian distributions. Hence the spread in the two directions may be described by spread functions of the form

$$
f=y \exp \left(-b \cdot \alpha^{2}\right)
$$




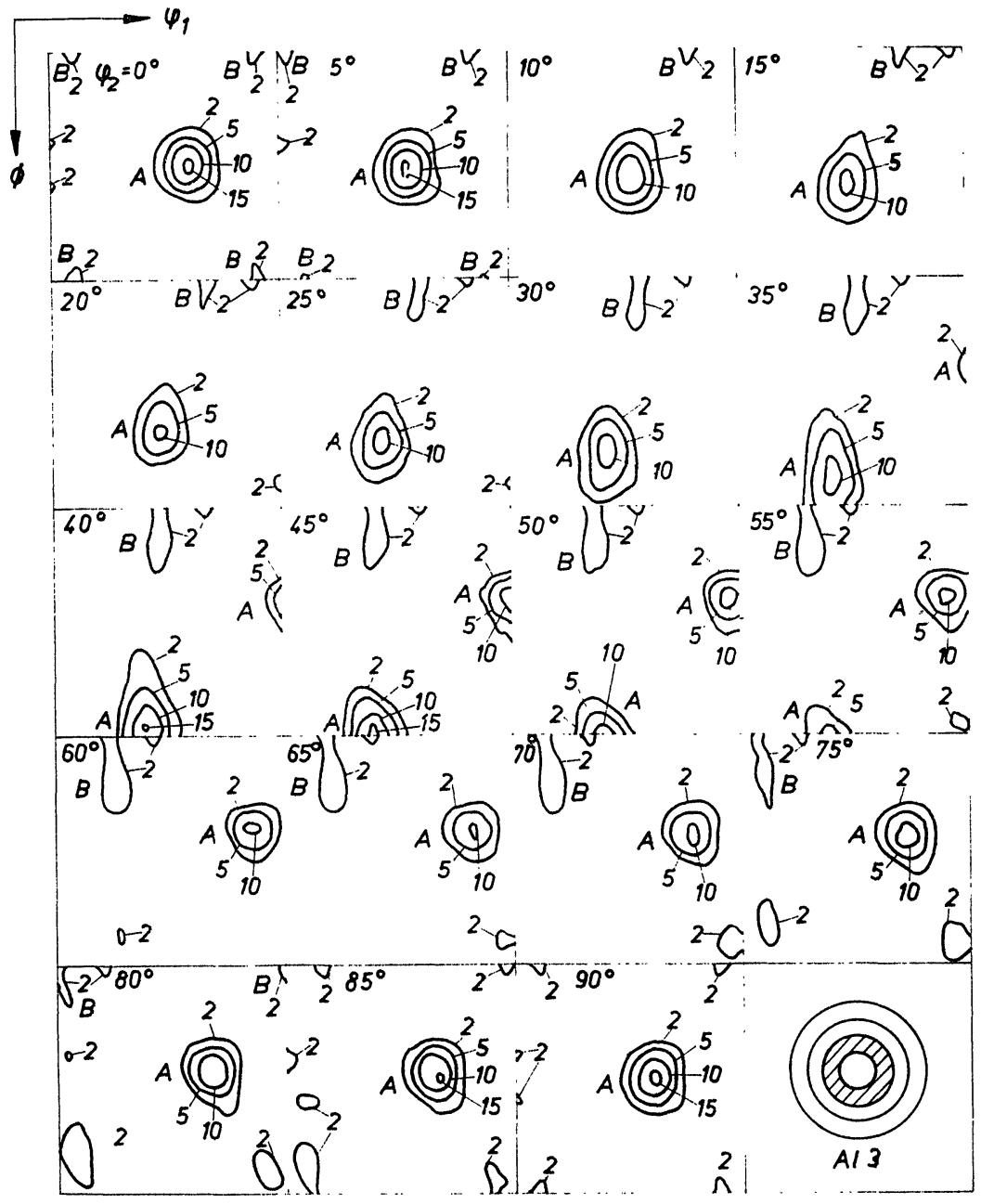

FIGURE $4 c$

FIGURE 4 Orientation distribution function for unwound cylinders taken from an aluminium wire cold drawn $97 \%$ in multiples of the random distribution.

(a) Al 1, outside diameter $=4 \mathrm{~mm}$.

(b) Al 2, outside diameter $=3 \mathrm{~mm}$.

(c) Al 3 , outside diameter $=2 \mathrm{~mm}$.

where $f$ is the orientation density at the angular distance $\alpha$ from the skeleton line, $y$ and $b$ are parameters characterizing the maximum orientation density and the slope of the spread respectively. Corresponding to Figure 8 directions of "wide" and "narrow" spread may be distinguished and characterized by two different slopes. As is seen from Figure 9 the narrow spread is nearly the same for the three different textures whereas the wide spread is different. It decreases continuously from the wire surface towards the centre and must be assumed to agree with the narrow spread at the very centre.
Interrelationship Between the Complete Orientation Distribution Function and the Inverse Pole Figure of the Wire Axis

Generally speaking the inverse pole figures of the different specimen directions as well as the pole figures of the different crystallographic directions are two-dimensional projections (in a generalized sense) of the three-dimensional orientation distribution function (see e.g. Ref. 10). For example the points of maximum orientation density of the different $\varphi_{2}$-sections (skeleton line) of Figure 4 were projected into one and the same point (namely 


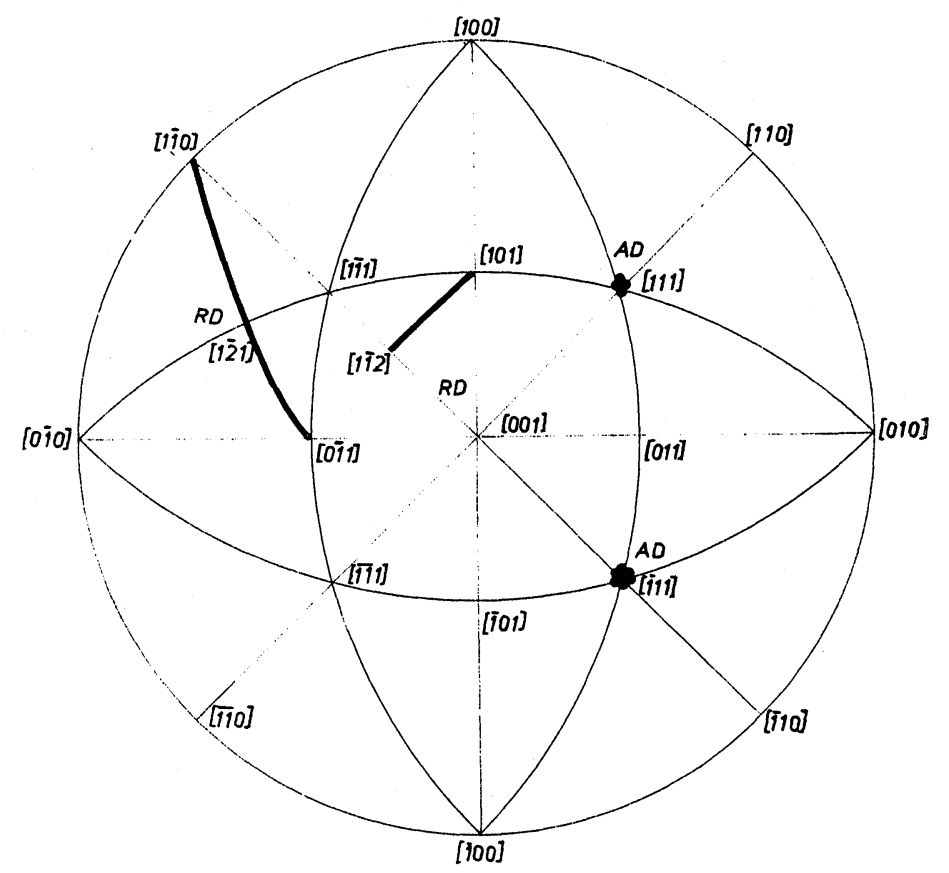

FIGURE 5 The orientations of maximum density (skeleton line) in stereographic projection. AD = axis direction, $\mathrm{RD}=$ radial direction.

(111)) of the inverse pole figure of the wire axis in Figure 5. Hence the spread contours of these sections about the maximum point are projected into spread contours about (111), all of which are superimposed thus forming the resulting spread contour of the inverse pole figure. Figure 10 illustrates in which way the narrow directions of the different $\varphi_{2}$-sections are superimposed and Figure 11 shows the same for the wide directions. Thus in the inverse pole figure a distribution of narrow and wide spread directions results which is schematically shown in Figure 12. In Figure 13 the inverse pole figures for the wire-axis direction along with those for the two other directions (radial and tangential directions) are shown. For the three unwound cylinder specimens $\mathrm{Al} 1$ to $\mathrm{Al} 3$ they were calculated from the three-dimensional orientation distribution functions with the help of the coefficients $C_{l}^{\mu \nu} .{ }^{10}$ The inverse pole figure of the centre of the wire, $\mathrm{Al} \mathrm{4}$, was calculated directly by the simpler fibre texture methods from (one-dimensional) pole figures. The inverse pole figure of the wire axis direction is a pure fibre texture quantity. It does not, per definition, contain any information about radial preferred orientation. The two other inverse pole figures do, however, contain information of this kind. If the texiures were ordinary fibre textures these two inverse pole figures ought to be identical which is, however, not the case. The inverse pole figures of the wire axis are of the type indicated schematically in Figure 12. They exhibit a narrow spread about (111) towards (110) and a wide spread towards (100). Contrary to the wide spread in the three-dimensional distribution, however, it is superimposed by a narrow spread and hence consists of two Gaussian components.

$$
f=y_{1} \exp \left(-b_{1} \cdot \alpha^{2}\right)+y_{2} \exp \left(-b_{2} \cdot \alpha^{2}\right)
$$

This is shown in Figure 14. The spread in the narrow direction, that is towards (110), is represented by one Gaussian component having the same slope as the narrow one of Figure 14. In Figure 15 the slopes of the wide spread components (occurring along (111)-(100)) and of the narrow ones (occurring along (111)-(100) and (111)-(110)) are shown for the textures corresponding to different distances from the wire surface. They agree reasonably well with those of Figure 9 in accordance with the above considerations.

\section{Interrelationship between the lnverse Pole Figure of} the Wire Axis and Fibre Texture Pole Figures

As was already mentioned the inverse pole figure of the wire axis is a true fibre texture quantity. It corresponds to a texture artificially symmetrized by 

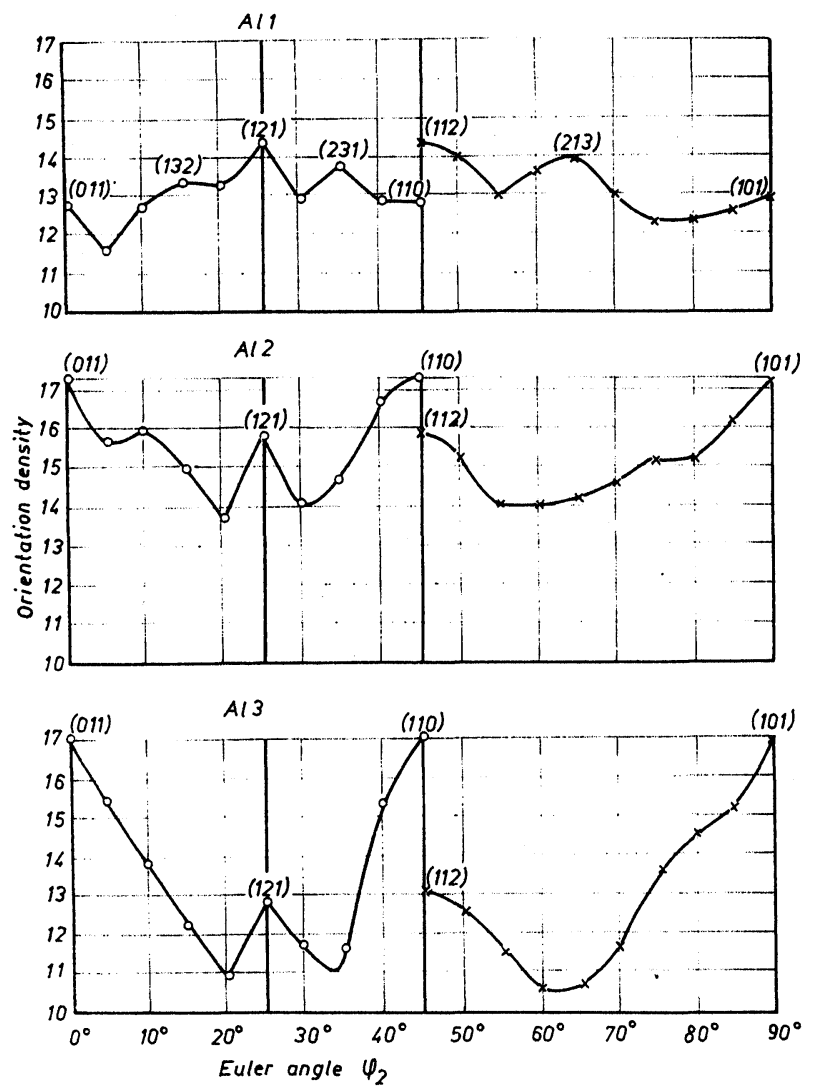

FIGURE 6 Orientation density along the skeleton line for three different distances from the wire axis.

a rotation about the wire axis. Hence it could be calculated already if the pole figures of Figure 2 were at first symmetrized that way, by rotating them about the wire axis, such as to yield onedimensional pole figures depending only on the angle towards the wire axis. Of course, pole figures of this type can be determined in a much simpler way by ordinary fibre texture methods without unwinding the cylinders, as it was done for the determination of the pole figures in Figure 2. For wire textures generally speaking the $(h k l)$-pole figure is obtained if the inverse pole figure of the wire axis is rotated about the $(h k l)$-direction. ${ }^{10}$ In the present case the intensity distribution of the (111)-pole figure in the vicinity of the wire-axis direction $\alpha=0$ corresponds to a rotation of the inverse pole figure about the (111)-direction. This is shown schematically in Figure 16. The pole figure, so to speak, is a one-dimensional "projection" of the two-dimensional inverse pole figure. It corresponds to an artificial symmetrization of the texture about the (111)-crystallographic direction. This sym- metrization must not be confused with the other one about the wire-axis direction which led from the three-dimensional orientation distribution function to the inverse pole figure. According to Figure 16 the pole figure is a certain mean value of the intensity distributions of the inverse pole figure taken along different $\beta$-sections. Hence it comprises a complicated mixture of wide and narrow spread components which does not allow in general to exactly determine the parameters of these two components separately. This is possible only with the help of the three-dimensional function or the inverse pole figure.

Nevertheless pole figure measurements can be used to qualitatively estimate the amounts of wide and narrow spread and hence to qualitatively estimate the spread parameters of the threedimensional distribution function, if its general features are known.

Besides this disadvantage inherent in pole figures there is, however, also an advantage with them, namely they are free of series truncation errors which influence the three-dimensional distribution function as well as the inverse pole figure, both of which are calculated with the help of a (truncated) series. In the present investigation the series were truncated at $l=22$. Hence according to an estimation given elsewhere ${ }^{10}$ the calculated functions are smeared out or artificially broadened with a half-maximum-width of the broadening function of about $5^{\circ}$. This is illustrated in Figure 17 which shows the course of the spread function along the wide and narrow directions of the inverse pole figure of $\mathrm{Al} 1$ compared with the corresponding (111)-pole figure. The broadening effect occurring in the inverse pole figure is clearly seen. It may be decreased by increasing the number of terms involved in the series development. This would, however, considerably increase the computer work required (in the present case, $l=22$, the series consisted of 125 terms).

\section{Fibre Texture Measurements}

Bearing in mind the relationships between the three-dimensional distribution functions, the inverse pole figures and the pole figures as well as the specific advantages and disadvantages of these functions certain parameters taken directly from pole figure measurements may be profitably used to get some qualitative information in a rather simple way. To this end ordinary fibre texture pole figures of the (111)-type were measured with several 
Al 1

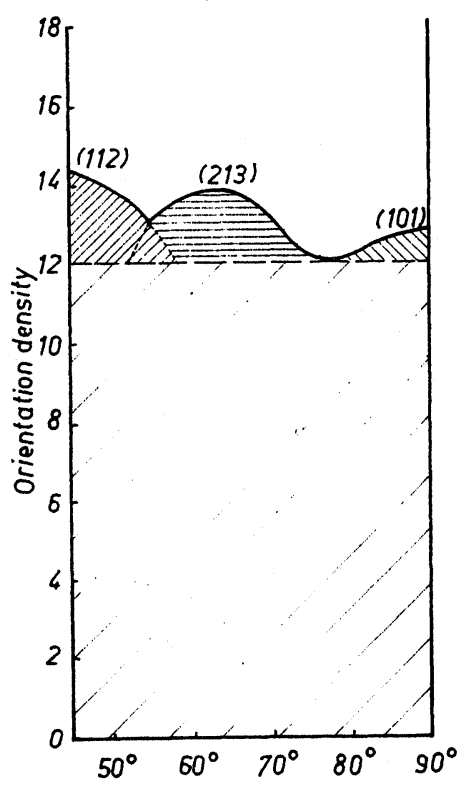

A/ 2

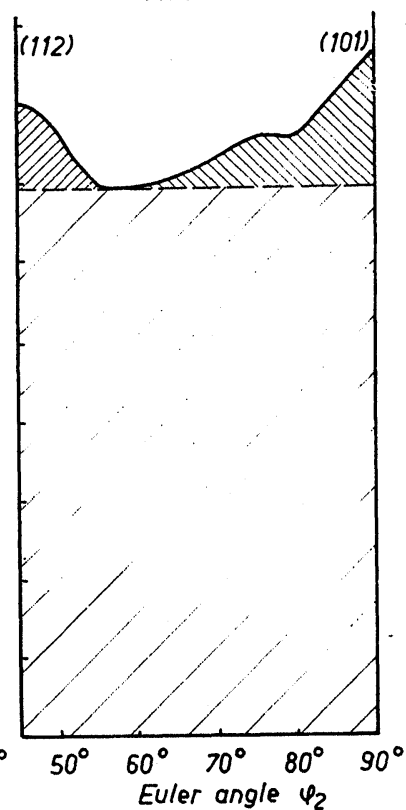

A/ 3

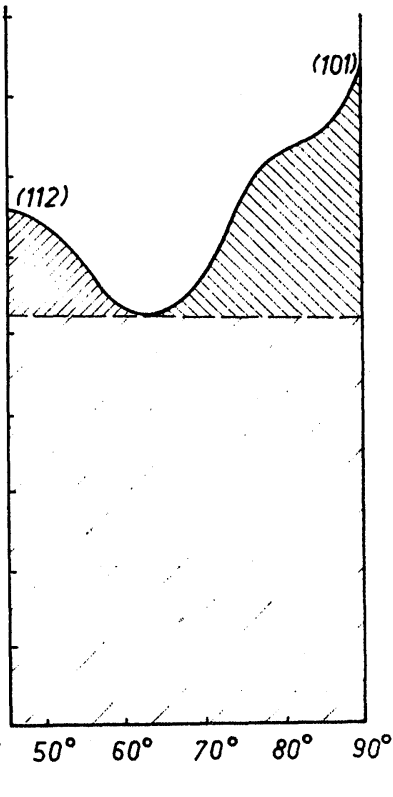

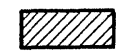

(112) [111]
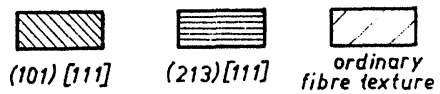

FIGURE 7 Estimation of the amount of cyclic and ordinary fibre texture components.
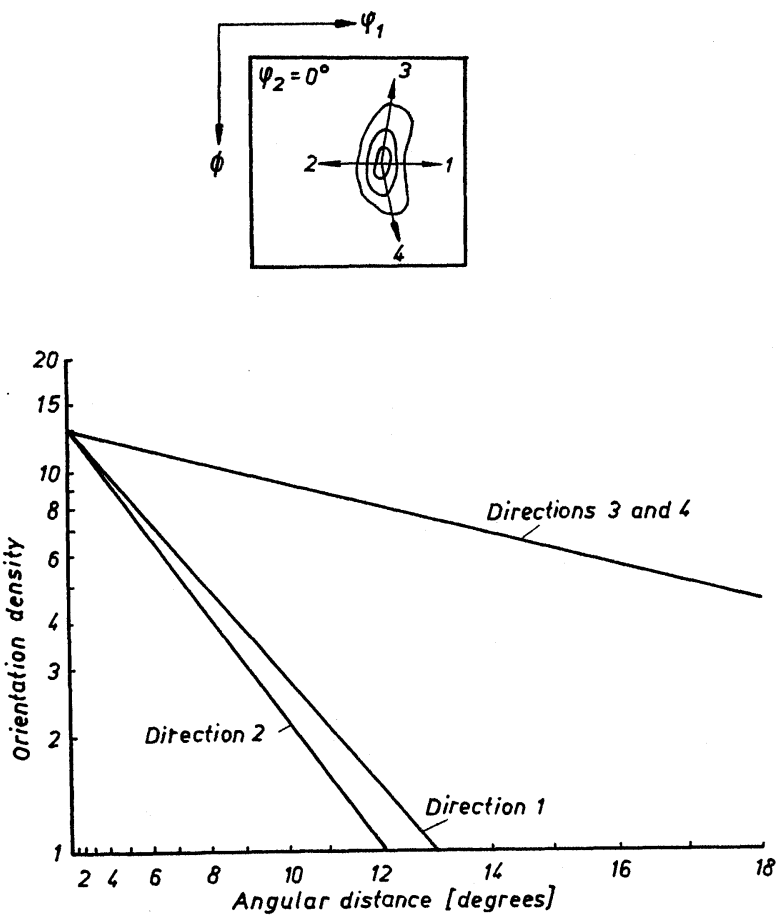

FIGURE 8 The spread about the point of maximum orientation density for the $\varphi_{2}=0^{\circ}$-section of the $\mathrm{Al} 1$. parameters varied. In the vicinity of the wire-axis direction they could be represented by a sum of two Gaussian components according to Eq. (2). In accordance with the above discussions this was not always exactly possible, but in these cases a best fitting approximation of the type of Eq. (2) could be used. The slopes of the two Gaussian components, the quantities $b_{1}$ and $b_{2}$, proved rather insensitive to variations of processing parameters. Hence the ratio $y_{1} / y_{2}$ could be used as a qualitative measure of the relative amount of wide spread in the texture.

In Figure 18 the dependence of this quantity on the diameter is shown for wires with different amounts of their outer layers removed either by machining or by etching. The first curve corresponds to the $4 \mathrm{~mm}$ wires used in the investigations described above and the second one is due to $0.18 \mathrm{~mm}$ wires investigated by X-ray diffraction. The results are to be compared with those from the unwound cylindrical specimens although in this case the inner parts of the wires were not removed. In accordance with the above results, in the innermost parts of the wires the wide spread component is almost completely absent; towards the surface it increases. There is, however, a discontinuity at 

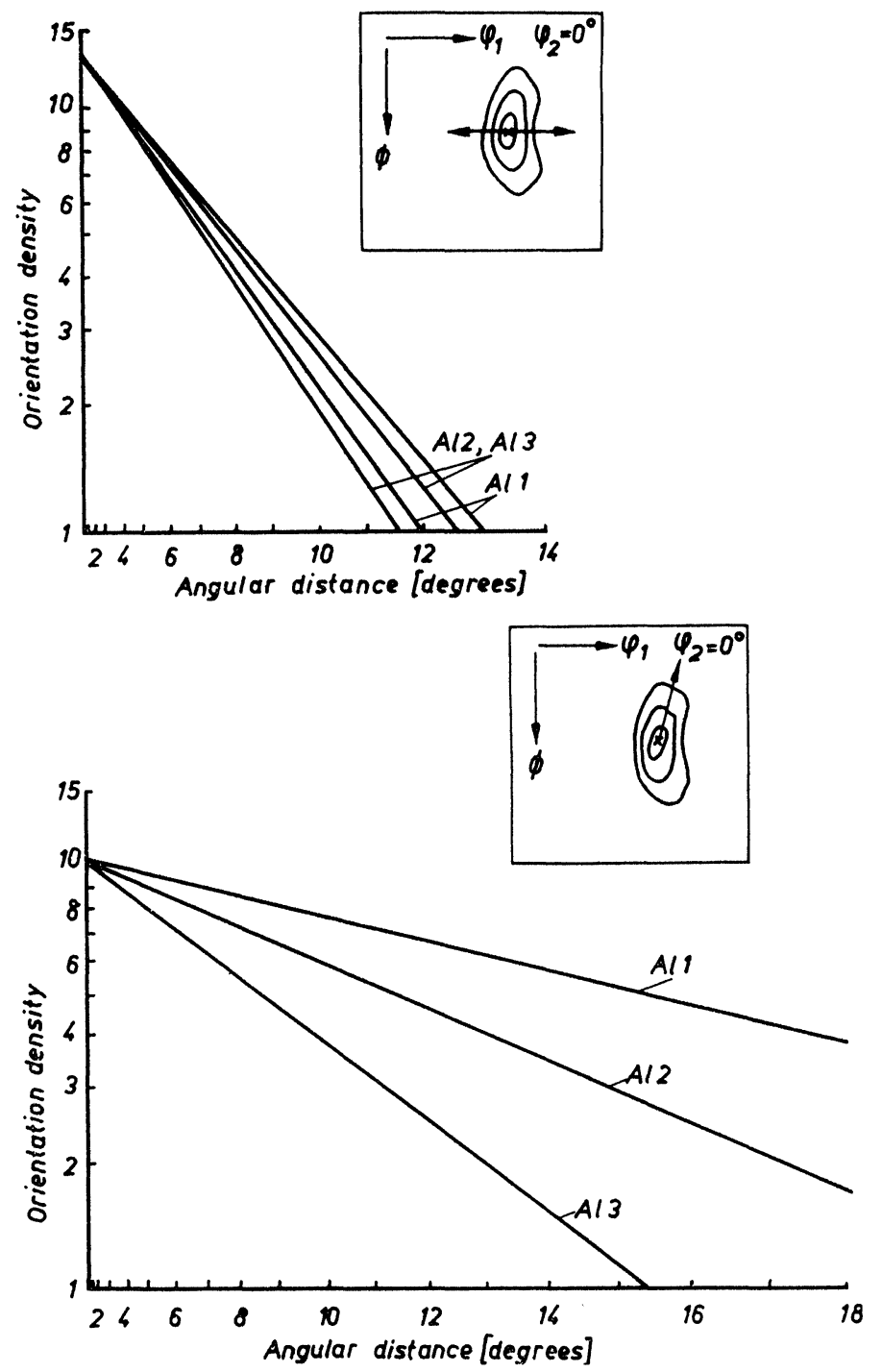

FIGURE 9 The slopes of the spread in the "narrow" and the "wide" directions respectively for different distances from the wire surface.
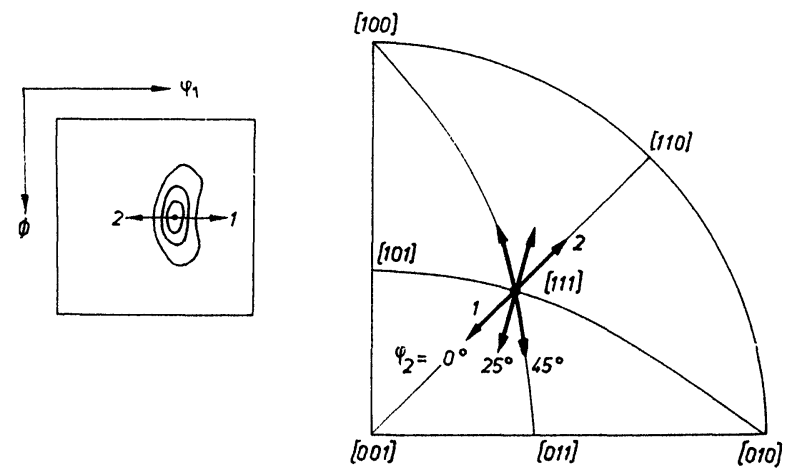

FIGURE 10 The images of the narrow spread directions of the different $\varphi_{2}$-sections in the inverse pole figure of the wire axis. about half the outer diameter. Both wires were deformed to the same degree of cold reduction, namely $97 \%$, and were of very similar materials. Hence the different amounts of spread must be ascribed to the different wire diameters, or more generally speaking to the different geometrical conditions for the material passing the die.

In Figure 19 the amount of wide spread is shown as a function of the degree of cold reduction (with no surface layers removed) starting from $1 \mathrm{~mm}$ recrystallized wires. It is seen that the sharpening of the texture during the drawing process is superimposed by a strong intermediate increase of the wide spread components. 

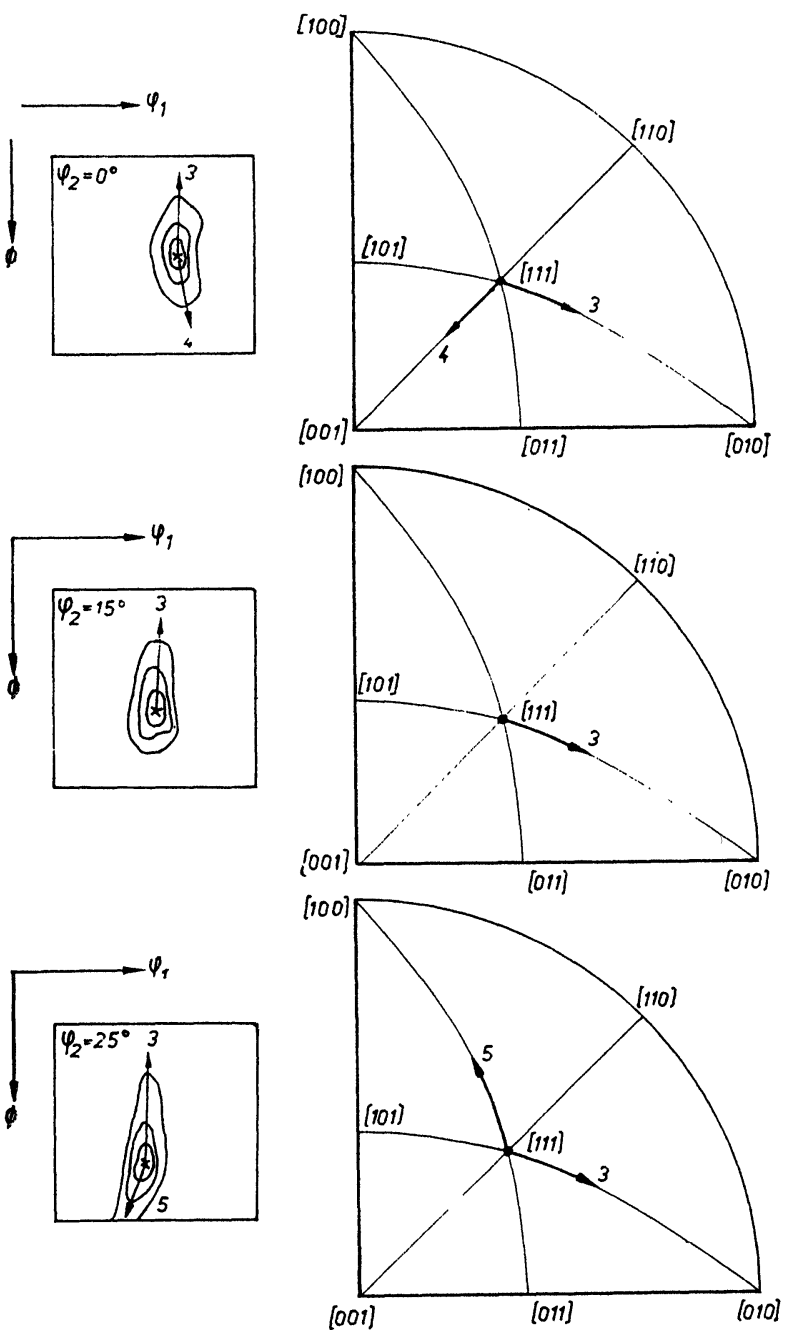

FIGURE 11 The images of the wide spread directions of the different $\varphi_{2}$-sections in the inverse pole figure of the wire axis.

\section{Recrystallization Textures}

All the results described so far corresponded to deformation textures. In addition to this some investigations were conducted on the recrystallization textures. Figure 20 shows the ratio $y_{1} / y_{2}$ as a function of the recrystallization temperature for different wires cold drawn from $1 \mathrm{~mm}$ to $0.1 \mathrm{~mm}$. Generally the amount of wide spread increases with increasing temperature. Furthermore, there were pronounced differences between the wires of the different charges which must be ascribed to differences in composition. It was, however, not the objective of the present investigation to pursue these questions in more detail. The

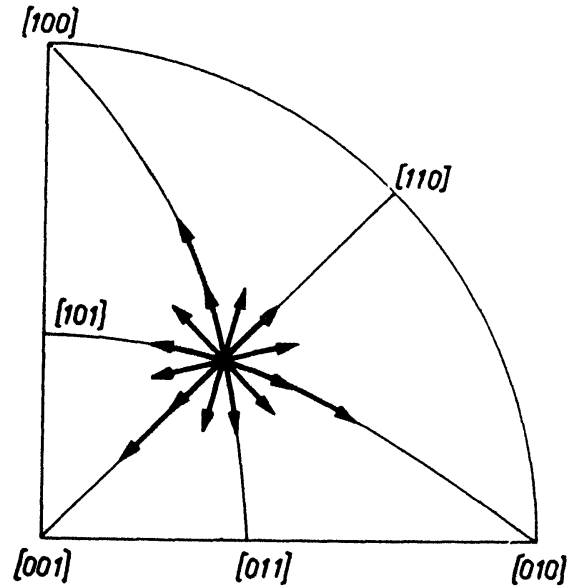

FIGURE 12 The resulting spread in the inverse pole figure of the wire axis (schematically).

recrystallization process being completed, prolonged annealing had no further influence on the shape of the distribution curve as is seen in Figure 21. A most pronounced effect on the shape of the distribution curve is being brought about, however, by the degree of cold deformation prior to recrystallization. This is shown in the second curve in Figure 19.

As is seen from this figure in the range of medium deformation degrees the amount of wide spread is much larger than that of the narrow spread component. These textures are considerably different from the previously described ones as is seen from the inverse pole figure shown in Figure 22. They contain a major part of (100)-component. Finally in Figure 23 the distribution of the spread components over the cross-section of the wires was investigated. It is different from the one in the cold worked state but it exhibits a discontinuity in the same range at about half the outer diameter.

\section{DISCUSSION}

As was mentioned earlier, the textures of unwound cylinders taken out of wires are expected to be intermediate between fibre textures and rolling textures because they are being brought about by deformations intermediate between the axially symmetric flow at the centre of a wire and plane strain deformation which approximately describes the rolling process. It is to be assumed that the deformation in a wire will be the more rolling-like the nearer to the surface. Hence the textures nearer 
$A D$

A19

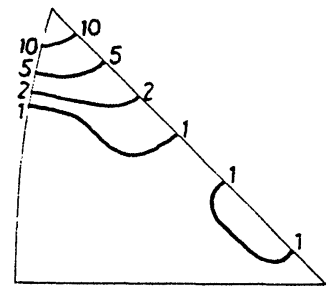

Al 2

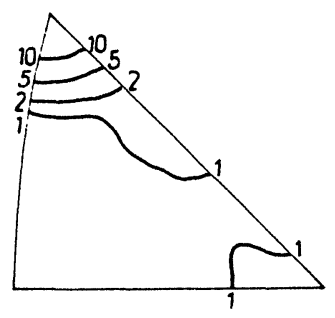

Al3
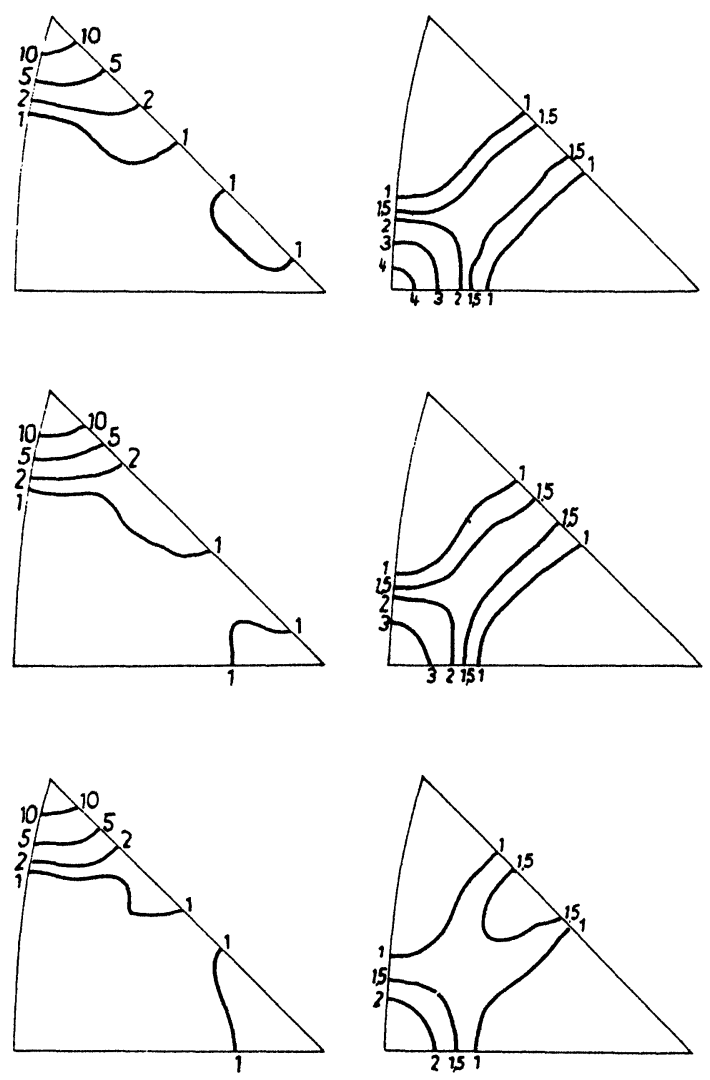

$R D$
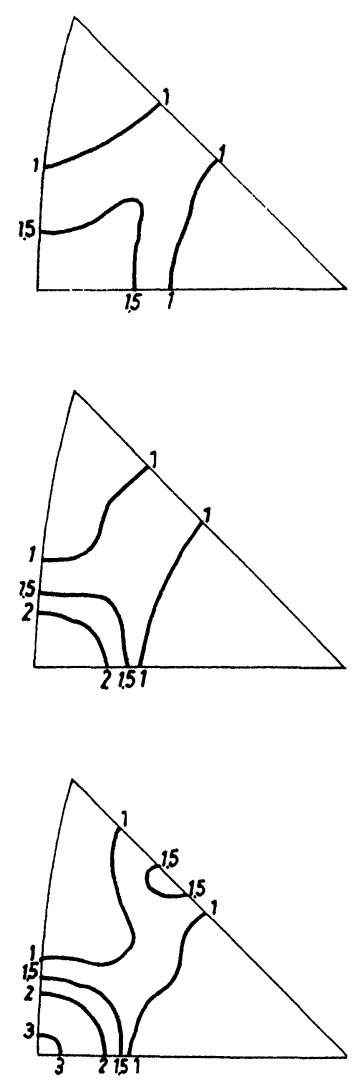

Al4

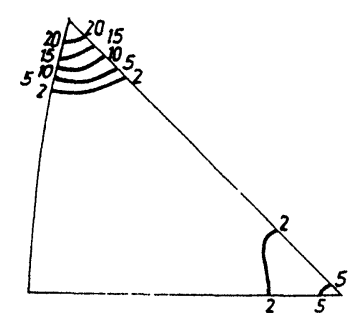

FIGURE 13 The inverse pole figures of the wire-axis direction, radial and tangential directions.

to the surface are expected to be closer to the rollingtype texture. The rolling texture of aluminium has not yet been studied in the three-dimensional orientation distribution function. It is, however, expected (at least to a first approximation) to be similar to that of copper. In Figure 24 the skeleton line of a $90 \%$ cold rolled copper sheet is shown. ${ }^{11}$ It is seen that the orientation of the normal direction is very similar to that of the radial direction in Figure 5.
The rolling direction on the other hand is to be compared with the wire-axis direction. It changes from a path in the vicinity of (111) in the case of rolling to the exact point of (111) in the case of wire drawing. This is in accordance with theoretical calculations on the basis of the Taylor theory. In Figure 25 the theoretical skeleton lines are shown for different values of the ratio of radial to tangential contraction. (They correspond to the skeleton 


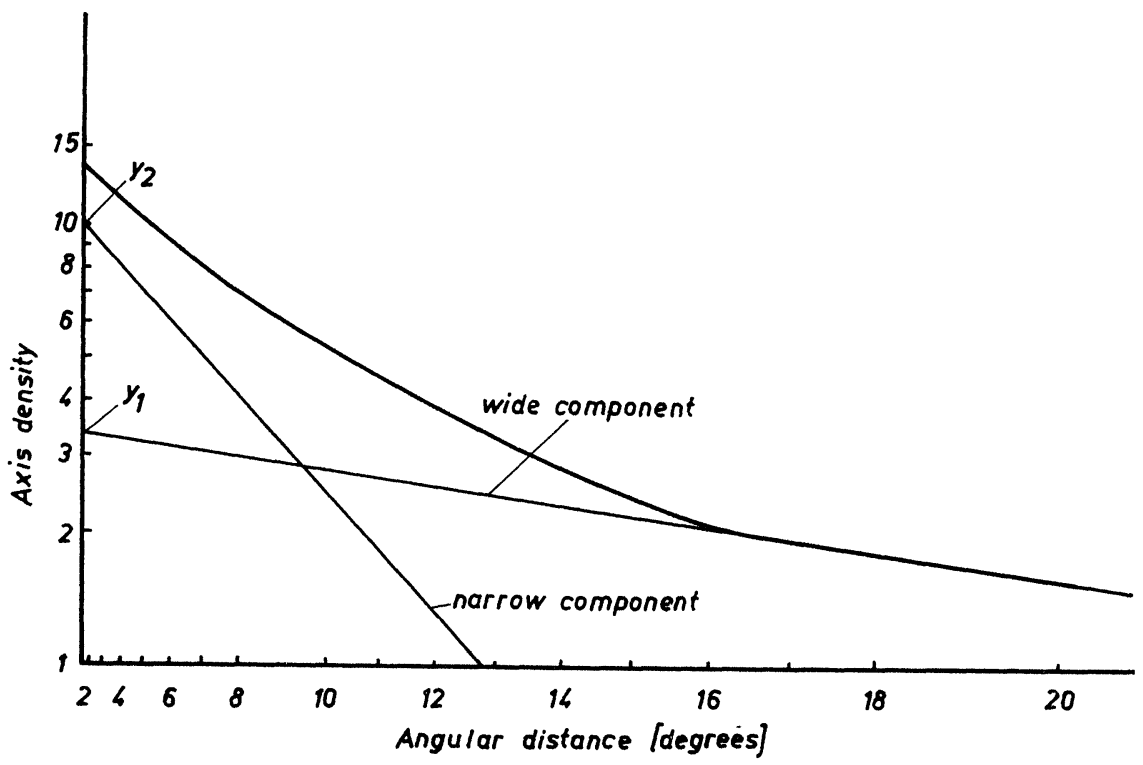

FIGURE 14 The spread along (111)-(100) in the inverse pole figure of the wire axis, consisting of two components.

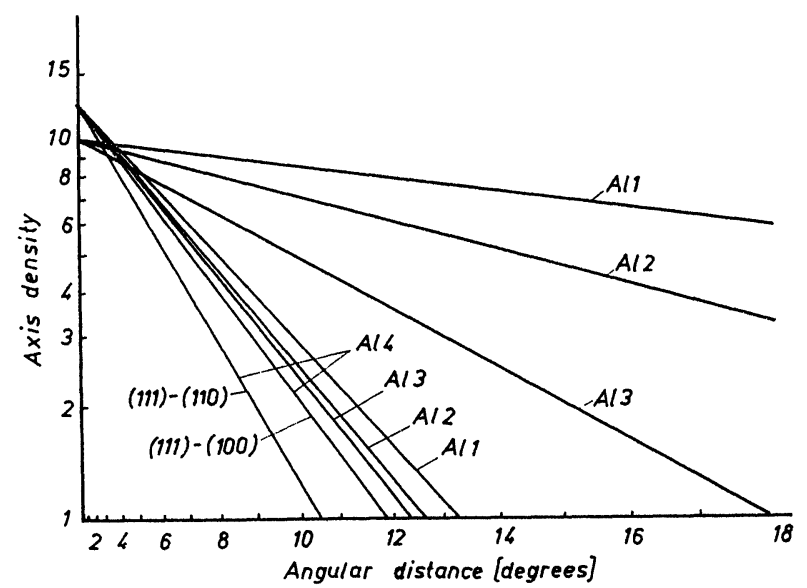

FIGURE 15 The slopes of the narrow and wide spread components in the inverse pole figures of the wire axis.

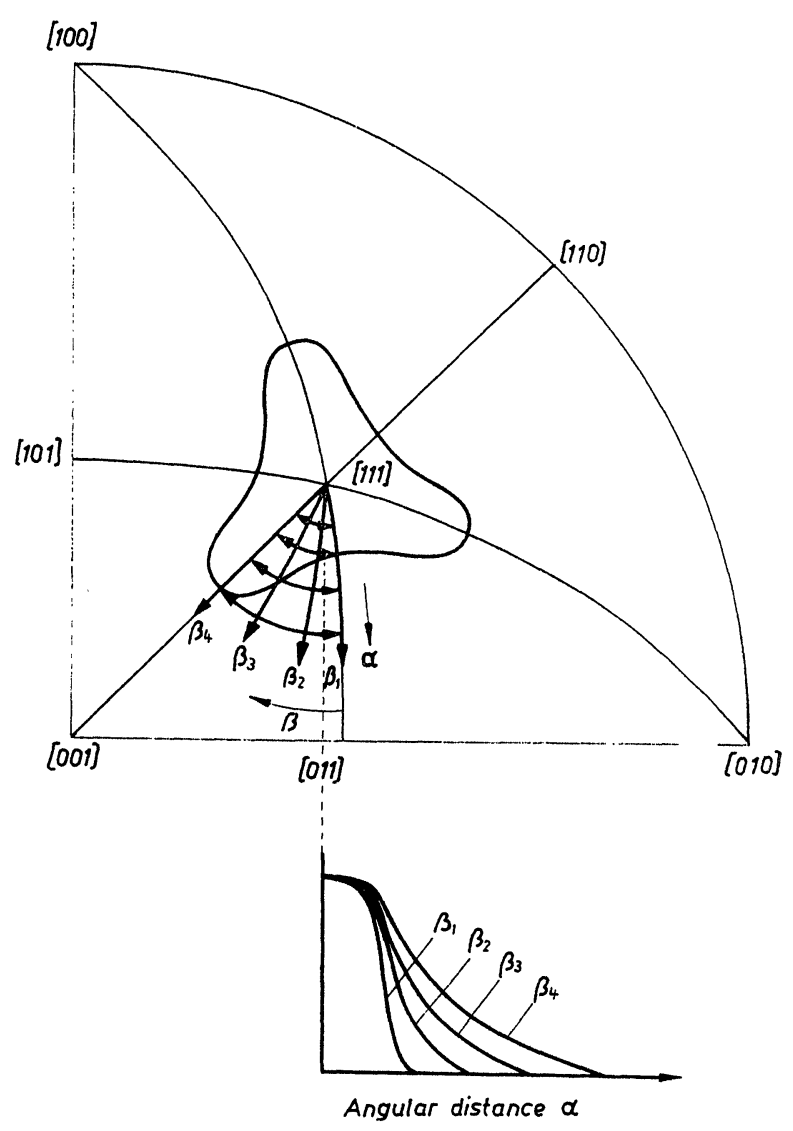

FIGURE 16 The mean values of the inverse pole figure taken along circles at the angular distance $\alpha$ from $h k l$ correspond to the values of the $(h k l)$-pole figure at the angle $\alpha$. 


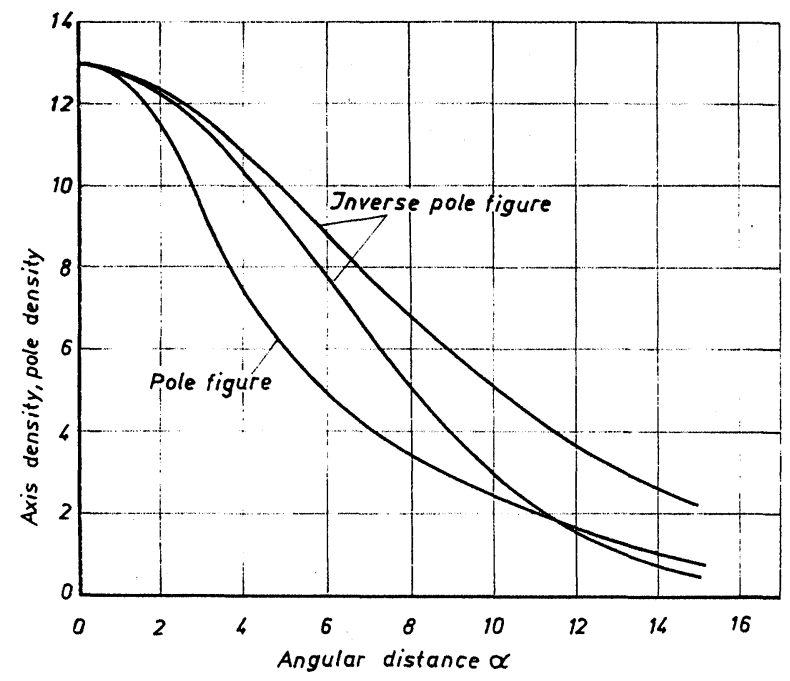

FIGURE 17 The (111)-fibre texture pole figure obtained by rotationally symmetrizing the (111)-pole figure of Figure 2 about the wire-axis direction and the sections along the wide and narrow spread directions of the inverse pole figure of $\mathrm{Al1}$, the outermost part of the wire.

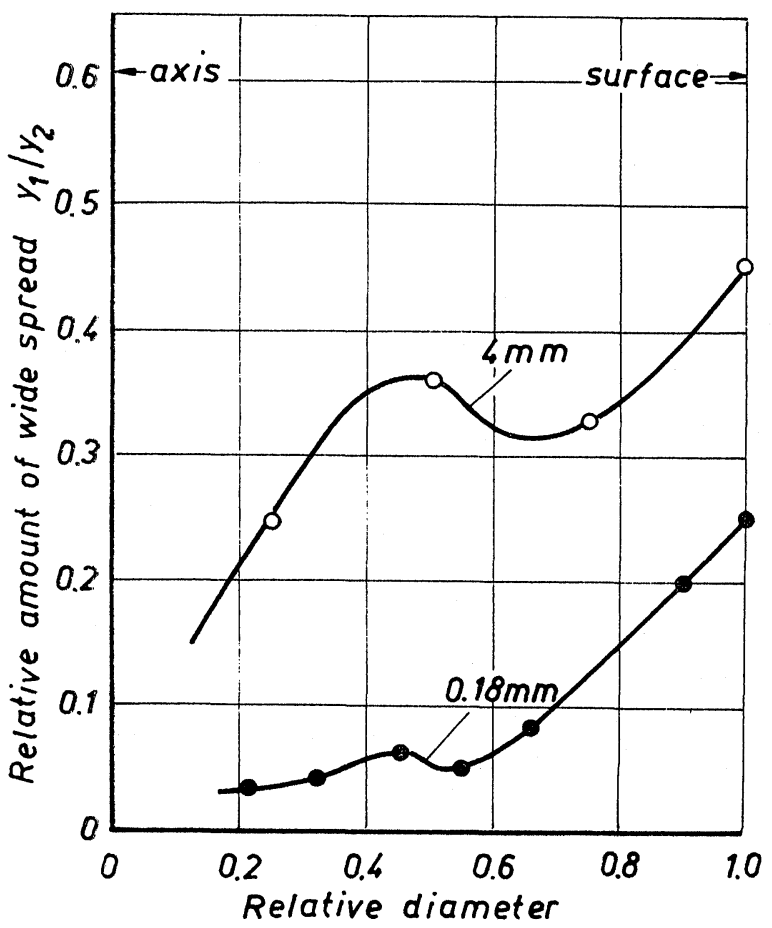

FIGURE 18 The relative amount $y_{1} / y_{2}$ of the wide spread component for cold drawn wires with their outer layers removed.

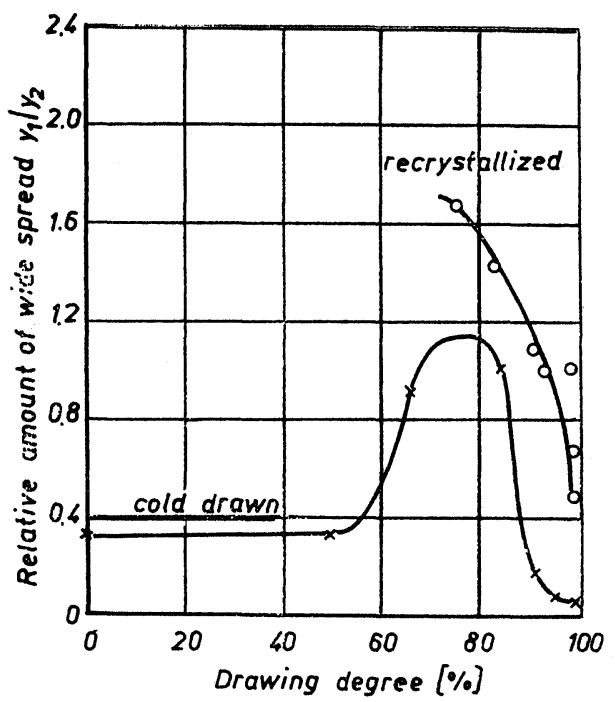

FIGURE 19 The relative amount $y_{1} / y_{2}$ of the wide spread component as a function of the degree of cold drawing for the cold drawn state [7] and recrystallized $30 \mathrm{~min}$ at $350^{\circ} \mathrm{C}$.

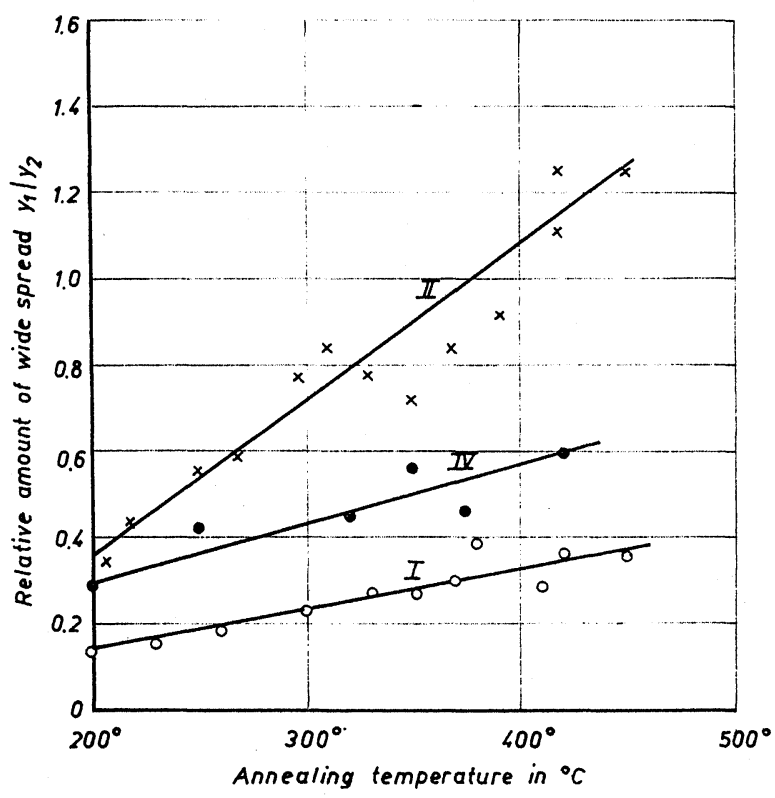

FIGURE 20 The ratio $y_{1} / y_{2}$ as a function of the annealing temperature for aluminium wires of different charges cold drawn from $1 \mathrm{~mm}$ to $0.1 \mathrm{~mm}$. 


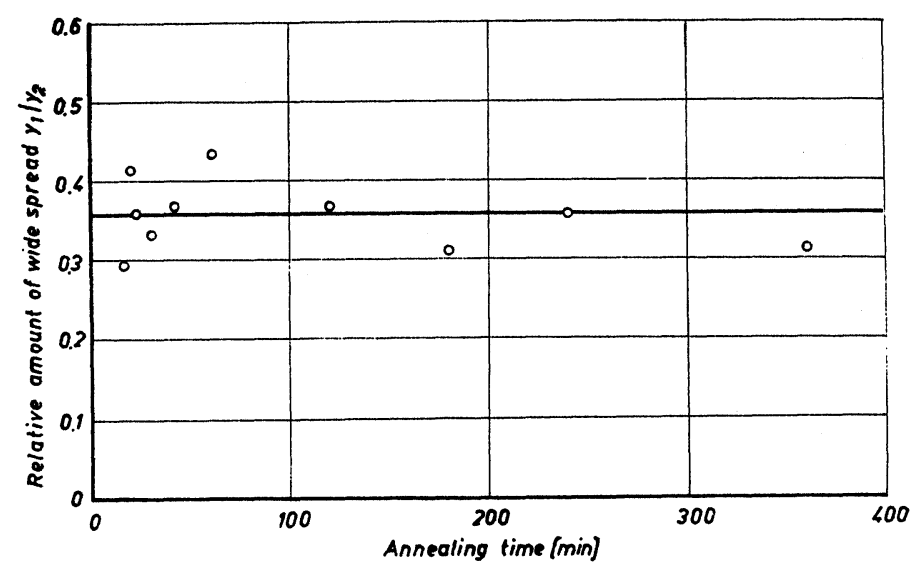

FIGURE 21 The ratio $y_{1} / y_{2}$ as a function of annealing time for $0.1 \mathrm{~mm}$ wires of charge II annealed at $250^{\circ} \mathrm{C}$.

FIGURE 22 Inverse pole figure of an aluminium wire cold drawn $70 \%$ recrystallized $30 \mathrm{~min}$ at $400^{\circ} \mathrm{C}$.
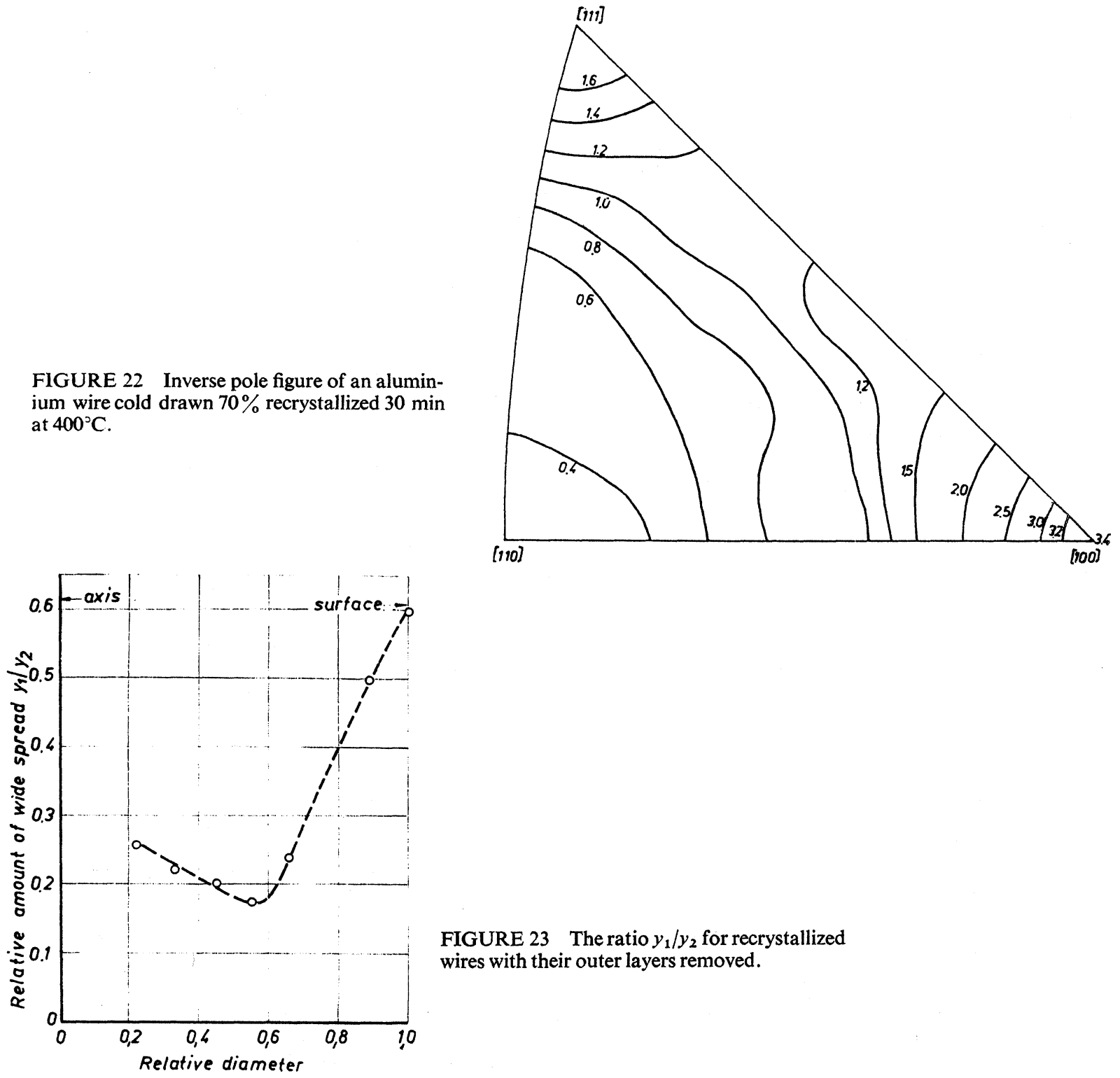

FIGURE 23 The ratio $y_{1} / y_{2}$ for recrystallized wires with their outer layers removed. 


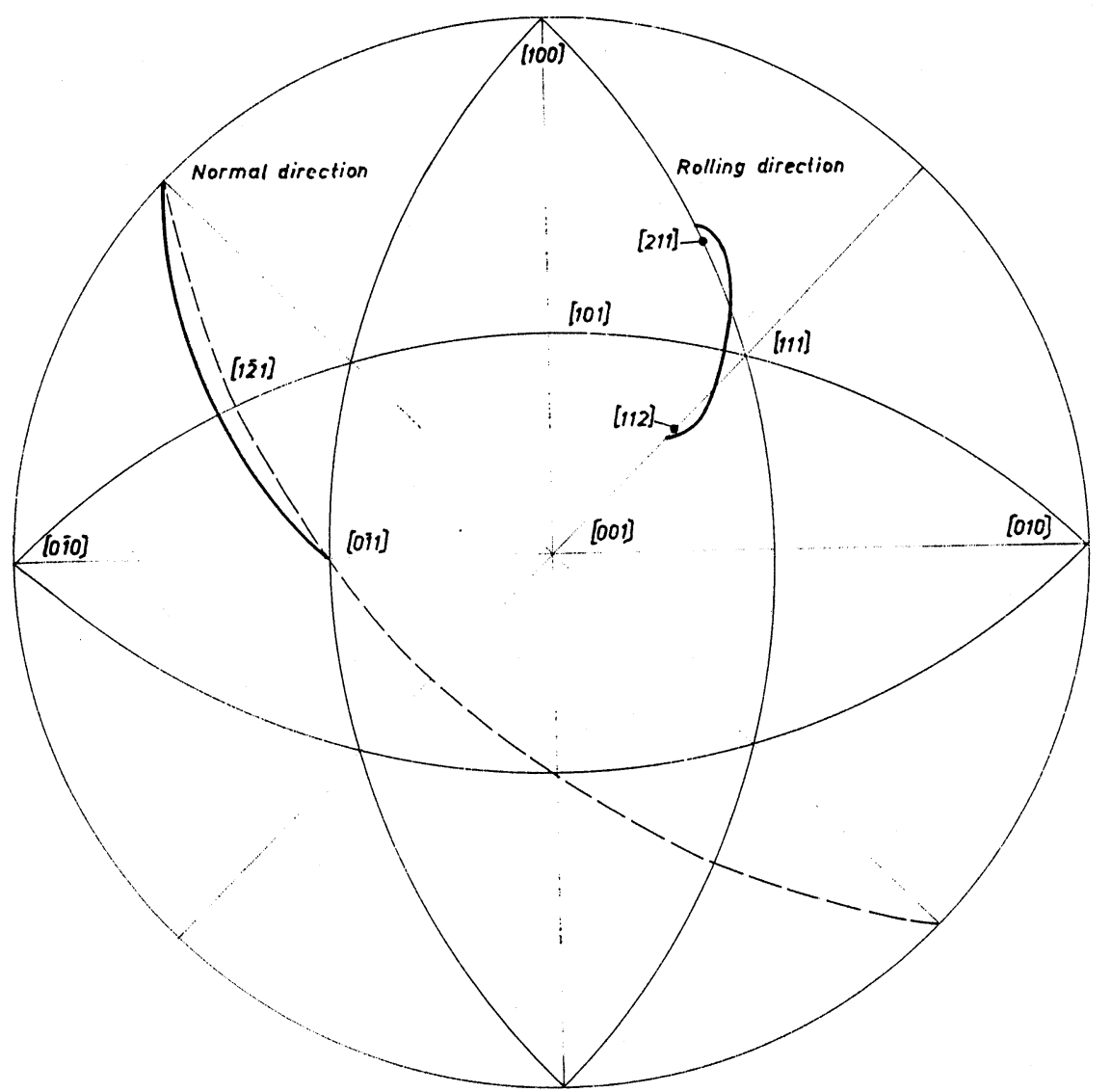

FIGURE 24 The skeleton line for a $90 \%$ cold rolled copper sheet.

lines for the same amount of lateral broadening for the b.c.c. case after exchanging normal and rolling directions. ${ }^{12}$ )

In Figure 26 the intensity along the skeleton line for the rolling texture is shown. It exhibits a maximum at the orientation (112)[111] that is at the $\varphi_{2}=25^{\circ}$-point of the skeleton line whereas at the $\varphi_{2}=0^{\circ}$-point it is minimum. Compared to this the texture of the innermost cylinder Al 3 in Figure 6 shows the opposite behaviour. The textures of $\mathrm{Al} 2$ and $\mathrm{Al} 1$ are intermediate to both types. In Figure 27 the difference of the orientation densities at $\varphi_{2}=25^{\circ}$ and $0^{\circ}$ is plotted as a function of the depth beneath the wire surface and for the rolling texture. This figure clearly shows the change of the wire texture towards the rolling-type if one proceeds from the interior of the wire towards its surface.

Another feature of the drawing textures investigated is the increase of the amount of wide spread towards the wire surface which manifests itself in the slopes of the wide spread curves of Figures 9 and 15 and in the course of the curves in Figure 18. It may be assumed that this spread is due to a corresponding variation of the flow direction for a volume element along its path through the die.

In order to quantitatively correlate this spread with the geometry of flow the drawing direction must be distinguished from its counter direction which was not possible in the present investigation because of the reasons mentioned above. Furthermore, for a quantitative comparison of the experimental results with theoretical calculations to be possible the actual deformation geometry ought to be determined. Thus in the present state only qualitative assessments can be made. Figure 18 shows that there is a discontinuity at about half the outer diameter which is in accordance with similar results by Linßen, Mengelberg and Stüwe. ${ }^{1}$ As is 


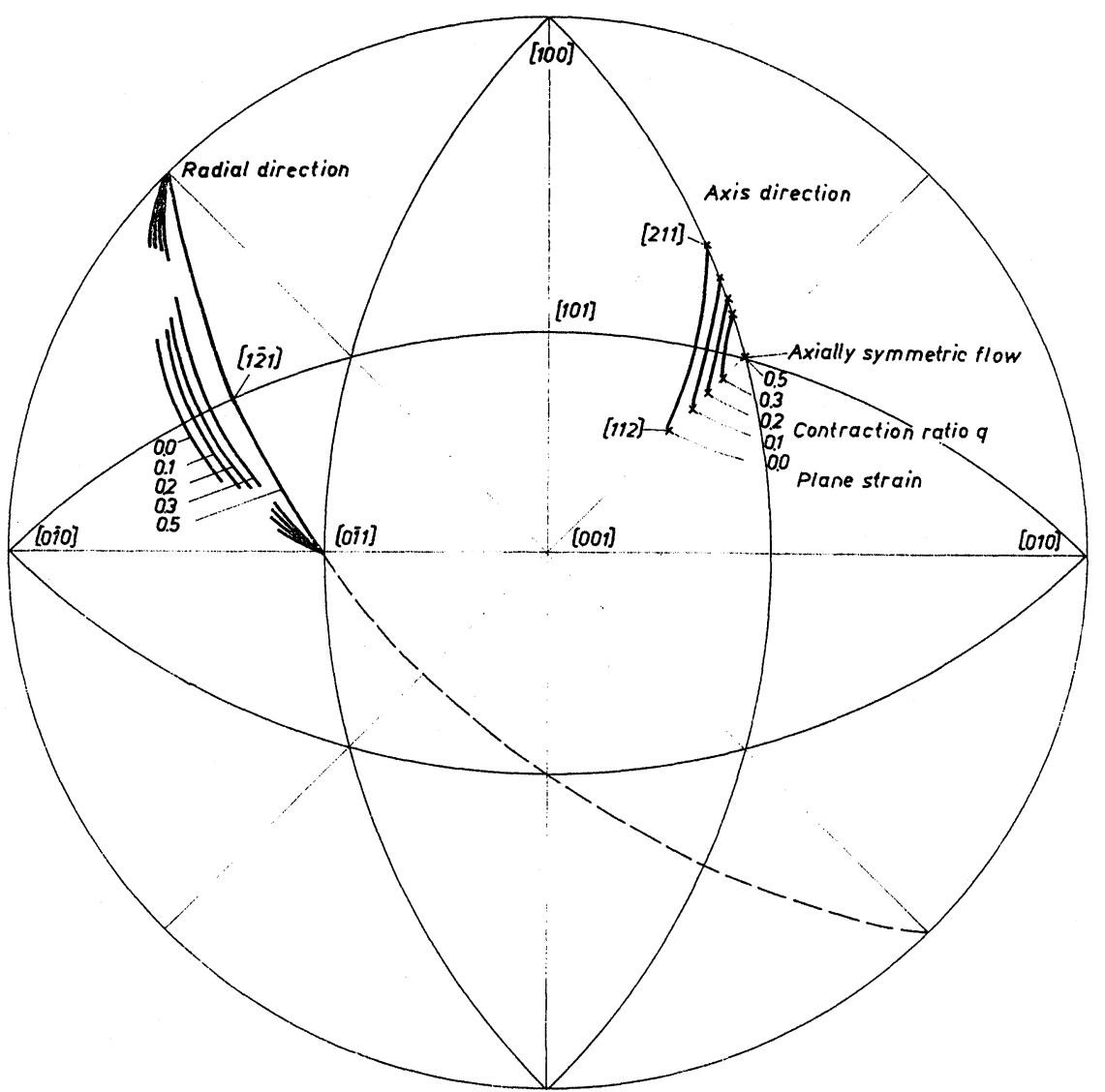

FIGURE 25 Theoretical skeleton lines for different ratios of radial to tangential contraction after [12].

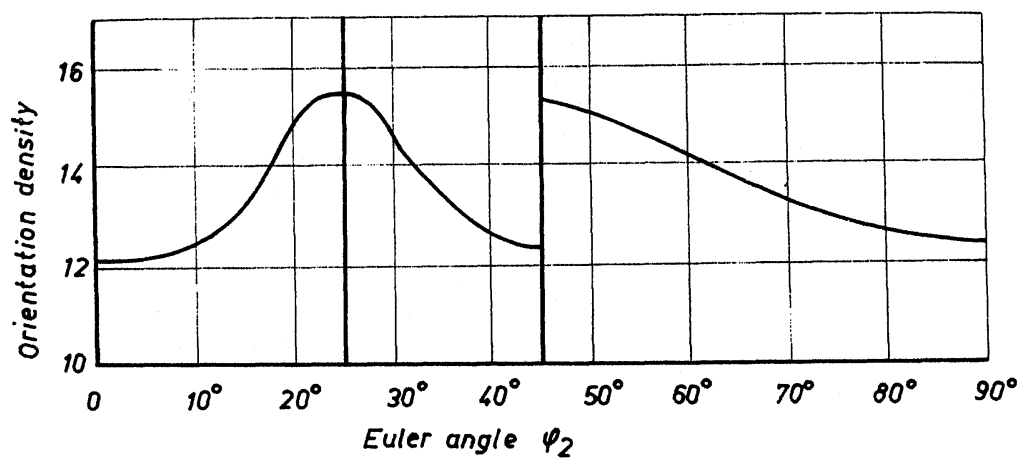

FIGURE 26 Orientation density along the skeleton line for a 95\% cold rolled copper sheet [13]. 


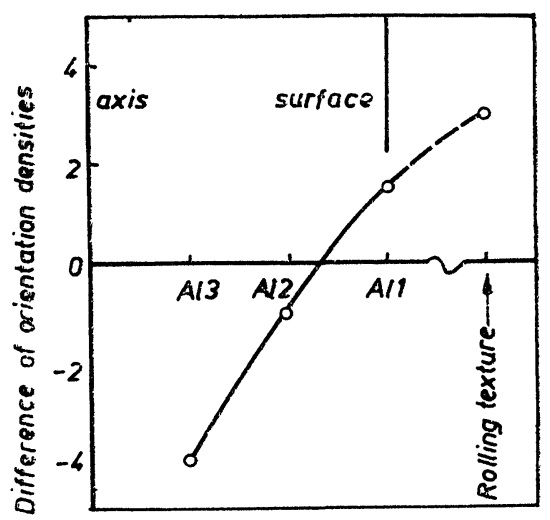

FIGURE 27 The difference of the orientation densities at the skeleton line for $\varphi_{2}=25^{\circ}$ and $\varphi_{2}=0^{\circ}$ for different distances from the wire surface and for the rolling type texture.

seen in Figure 4, in addition to the main texture components labelled $\mathrm{A}$ there is a second, much weaker one labelled B which was, however, only scarcely above the limit of error. It corresponds to orientations having a (100)-direction approximately parallel to the wire axis, and hence to the (100)-component of true fibre textures. This component is the strongest at the centre of the wire which is more clearly seen from the inverse pole figures in Figure 13.

In Figure 19 the dependence of spread on the degree of cold deformation is shown. It exhibits a strong increase at an intermediate range of about $50-80 \%$ reduction. In this range the orientations of a certain amount of crystallites must have moved away from the stable orientation (111), whereas at higher as well as lower deformations, they move toward this orientation. For high degrees of deformation it shows a sharp decrease in this range; and this was also shown by the corresponding inverse pole figures. ${ }^{7}$ For very low degrees of deformation i.e. $\leqq 1 \%$ an orientation change of the crystallites towards (111) was found too. ${ }^{14}$ The orientation change towards (111) is in quantitative agreement with calculations based on Taylor's theory of polycrystal plasticity. ${ }^{12}$ Hence Figure 19 shows that at intermediate degrees of deformation a different mechanism must have been operative. Similar results have been obtained for the rolling textures of copper. ${ }^{13,15}$ Since twinning as a deformation mechanism is unlikely to occur in aluminium it may be assumed that anisotropic hardening which is known to occur at intermediate degrees of deformation may be involved.
As is seen from Figure 19, limited results on the recrystallization textures showed a strong dependence on the corresponding deformation textures. The sharper the deformation texture is the sharper is the recrystallization texture. In general, however, the latter contains a larger amount of wide spread. A similar relation between drawing and recrystallization textures also holds for the dependence on the distance from the wire surface, Figure 23. These results may be understood in terms of an oriented nucleation theory. In a former investigation the mean grain sizes of crystals in different orientations have been determined. ${ }^{16}$ They were found to be independent of the angular distance from the preferred (111) orientation. Hence it was concluded that the larger volume fraction of crystals having approximately (111) orientation was due to a larger number of nuclei in this orientation rather than to preferred growth of randomly oriented nuclei. Similar results have been obtained recently by Ferran, Doherty and $\mathrm{Cahn}^{17}$ who investigated the orientations of new grains formed by recrystallization of aluminium. In this sense it must be concluded from Figure 19 that the wide spread component of the drawing texture preferentially contributes to nucleation.

\section{REFERENCES}

1. G. Linßen, H. D. Mengelberg and H. P. Stüwe, $Z$. Metallkunde 55, 600 (1964).

2. H. Aernould, J. Kokubo and H. P. Stüwe, Z. Metallkunde 57, $217(1966)$.

3. J. Tobisch, M. Betzl and P. Reichel, Exp. Techn.d. Physik 17, 391 (1969).

4. M. Betzl and K.H. Kleinstück, Kernenergie 4, 923 (1961).

5. K. H. Kleinstück and J. Tobisch, Kristall und Technik 3, 455 (1968).

6. H.J. Bunge, $Z$. Metallkunde 56, 38 (1965).

7. H. J. Bunge, Mber. Dtsch. Akad. Wiss. 5, 293 (1963).

8. H. J. Bunge, Kristall und Technik 6, 429 (1971).

9. U. Schläfer, Thesis Bergakademie Freiberg (1971).

10. H. J. Bunge, Mathematische Methoden der Texturanalyse (Akademieverlag, Berlin, 1969).

11. H. J. Bunge and J. Tobisch, Z. Metallkunde 59, 471 (1968).

12. H. J. Bunge, Kristall und Technik 5, 145 (1970).

13. H. J. Bunge, J. Tobisch and W. Sonntag, J. Appl. Cryst. 4, 303 (1971).

14. H. J. Bunge and R. Fuchs, Phys. Stat. Sol. 32, 169 (1969).

15. W. Truszkowski and J. Król, Mem. Sci. Rev. Met. 65, 907 (1968).

16. H. J. Bunge, Z. Metallkunde 58, 649 (1967).

17. G. L. Ferran, R. D. Doherty and R. W. Cahn, Acta Met. 19, 1019 (1971). 